\title{
Research on the GARCH model of the Shanghai Securities Composite Index
}

\author{
Dancheng Luo \\ School of Economics \\ Shenyang University of Technology \\ Shenyang, China \\ donna_luo@sina.com
}

\author{
Yaqi Xue \\ School of Economics \\ Shenyang University of Technology \\ Shenyang, China \\ 1046084081@qq.com
}

\begin{abstract}
GARCH model can be used to describe the characteristics of returns fluctuation, which can forecast the returns and risk of financial assets. This paper makes use of GARCH model and its hybrid models, such as GARCH-M model, E-GARCH model, T-GARCH model and I-GARCH model, to the empirical research on the data of Shanghai Securities Composite Index from January 2, 2001 to May 20, 2013. The results show that there are the characteristics of High Kurtosis and Fat Tail, Volatility Clustering and the phenomenon of high risk and high reward.
\end{abstract}

Keywords- volatility; Arch model; Garch model

\section{INTRODUCTION}

The stock market is full of uncertainty, coupled with the increasing efficiency of information transfer. The stock price volatility is an effective tool to measure the market information. It reflects the activity level of the market while the fluctuation is within range, but if it is too much, the market will be risky. China, as an emerging country, the development of its capital market is not perfect, and also it is very typical for the stock market suffering from the highrisk high-return. After the split share structure reform, nontradable shares shift to tradable shares, further increases the volatility of stock returns. Therefore, it is a necessity for both investors and institutions to know more about the volatility of stock market returns.

This paper studied the ARCH effect and GARCH model and its development models of the wave characteristics on the econometric perspective, based on Shanghai index daily yield using stata10 software.

\section{THEORETICAL DISCOURSE}

\section{A. Arch Model}

$\mathrm{ARCH}$ model as autoregressive conditional heteroskedasticity model, is first proposed in "econometrics" magazine in 1982 by Professor Robert Engle of University of California, San Diego. The model described the variance sequence of interference terms in the form of autoregressive. ARCH models can be used to illustrate the autocorrelation characteristics of the volatility of the time series.

\section{B. Garch Model}

GARCH model is generalized autoregressive conditional heteroskedasticity model, proposed by T.Bollerslev in 1986 . Actually it is an expansion of ARCH model. Lag terms of the variance of disturbance term is added in the conditional variance equation on the basis of the $\mathrm{ARCH}$ model. Compared to ARCH model, GARCH model can better reflect the long-term memory characteristics of the actual data. So it has been widely used in the forecast of financial asset return and risk. It is noteworthy that any higher order $\mathrm{ARCH}$ process can be represented as a low-level GARCH process and empirical studies show that even the most simple GARCH $(1,1)$ model can usually achieve a good fitting effect.

\section{Garch-M Model}

GARCH-M model was proposed by Engle, Lillien and Robins in 1987. It describes the volatility of financial time series affect their yields by adding residual variance in mean equation. And it can be used to verify that whether there are the high-risk high-return characteristics in the financial products.

\section{E-Garch Model}

In your paper title, if the words "that uses" can accurately replace the word "using", capitalize the "u"; if not, keep using lower-cased. E-GARCH model makes a distinction of the two interferences by giving "positive interference" and "negative interference" different coefficient estimation. And it can overcome the defect that $\mathrm{ARCH}$ and GARCH model cannot distinguish the asymmetry effect of positive and negative impacts. So it can be used to describe the asymmetric impact of positive and negative interference in the stock market.

Conditional variance equation: $\operatorname{Var}\left(e_{-} t\right)=\operatorname{sigma}^{\wedge} 2 \_t$

$=\mathrm{g} \_\mathrm{t}$

$\log \left(g_{-} t\right)=b 0+c 1 *\left[\left|e_{-} t-1\right|-E\left|e_{-} t-1\right|+r h o * e_{-} t-1\right]$

$+\mathrm{c} 2 *\left[\left|\mathrm{e}_{-} \mathrm{t}-2\right|-\mathrm{E}\left|\mathrm{e}_{-} \mathrm{t}-2\right|+\right.$ rho $\left.^{*} \mathrm{e}_{-} \mathrm{t}-2\right]$

$+\ldots$

$+c p *\left[\left|e_{-} t-p\right|-E\left|e_{-} t-p\right|+\right.$ rho*e_t-p]

$+\mathrm{d} 1 * \log \left(\mathrm{g}_{-} \mathrm{t}-1\right)+\mathrm{d} 2 * \log \left(\mathrm{g}_{-} \mathrm{t}-2\right)+\ldots+\mathrm{dq} * \log \left(\mathrm{g}_{-} \mathrm{t}-\mathrm{q}\right)$

e_t is the residual of the mean equation. 1 <delta indicates that a positive interference will increase volatility, while negative interference will do the opposite; $0<$ delta $<=$ 1 indicates "good news" can cause larger fluctuations than "bad news"; delta $=0$ means both positive interference and 
negative interference can cause the same fluctuation; $1<=$ delta $<0$ indicates "bad news" can cause larger fluctuations than "good news"; delta $<1$ indicates a positive interference will reduce volatility, while negative interference will do the opposite.

\section{E. GJR-Garch vs T-Garch Models}

Be aware of the different meanings of the homophones "affect" and "effect", "complement" and "compliment", "discreet" and "discrete", "principal" and "principle". GJRGARCH vs T-GARCH modesl, is proposed by Glosten, Jagannathan, and Runkle in 1993 and Zakoian in 1994 respectively. It mainly distinguishes positive and negative interference by adding dummy variables in GARCH model.

Conditional variance equation: $\operatorname{Var}\left(\mathrm{e}_{-} \mathrm{t}\right)=\operatorname{sigma}^{\wedge} 2 \_\mathrm{t}$

$=\mathrm{g} \_\mathrm{t}$

$=\mathrm{b} 0+\mathrm{c} 1 * \mathrm{e}^{\wedge} 2 \mathrm{t}-1+\mathrm{d} 1 * \mathrm{~h} \mathrm{t}-1+\mathrm{f} 1 * \mathrm{e}^{\wedge} 2 \mathrm{t}-1 * \mathrm{I} \mathrm{t}-1$

e_t is the residual of the mean equation

I_t $-1=1$ (if e_t $-1>=0)$; I_t $-1=0$ (if e_t $-1<0$ )

If $\mathrm{f} 1<0$, indicates that "bad news" can cause larger fluctuations than "good news"; if f1>0, indicates that "good news" can cause larger fluctuations than "bad news"; if $\mathrm{f1}=$ 0 , T-GARCH model is equal to the ordinary GARCH model.

\section{F. I-Garch Models}

I-GARCH model adds constraints to coefficients of ARCH part and GARCH part of GARCH model in order to make the model the characteristics of long-term memory.

\section{EMPIRICAL RESEARCH}

\section{A. The Distribution Characteristics of the Shanghai}

Securities Composite Index Returns: High kurtosis and fat Tail, Volatility Clustering.

This paper's research object is the Shanghai securities composite index from January 2, 2001 to May 20, 2013. Based on the sequence diagram (Fig.1), we can already aware of the clustering phenomenon that strong fluctuations follow strong fluctuations and weak fluctuations follow weak fluctuations. This feature is more obvious in the sequence diagram (Fig.2) of returns and its absolute value.

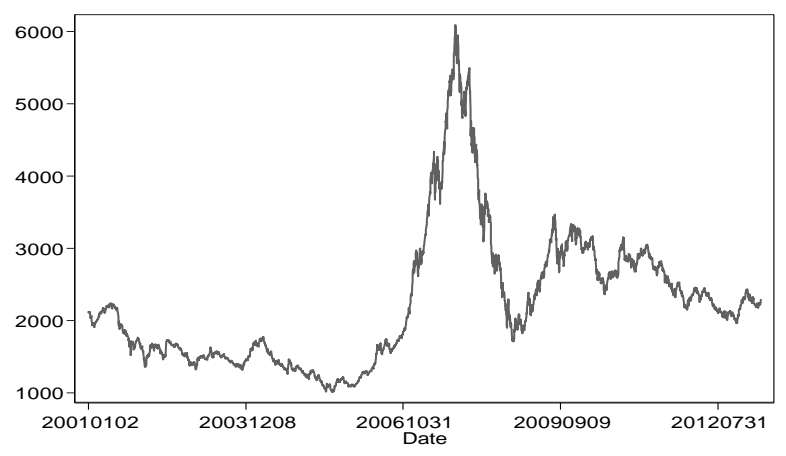

Figure 1. Sequence diagram of Shanghai securities composite index

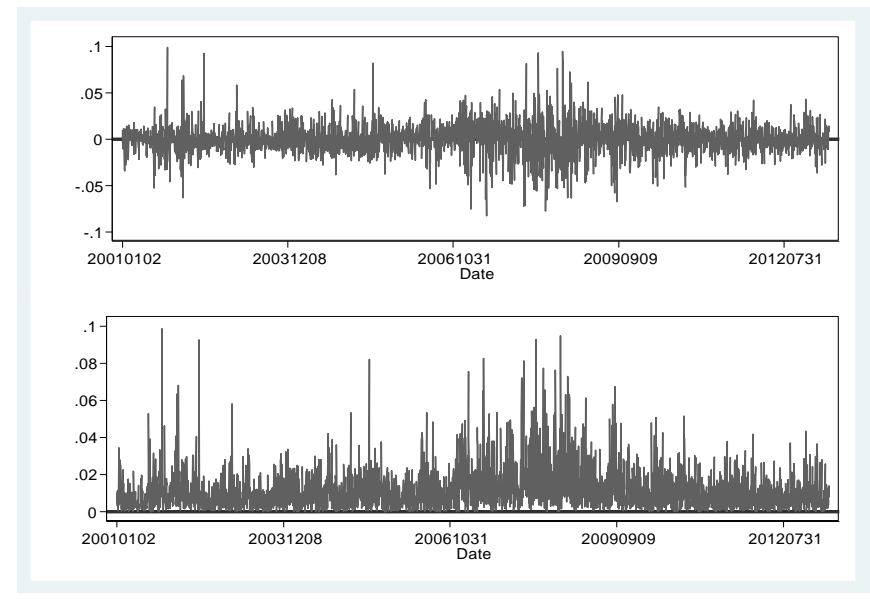

Figure 2. Sequence diagram of returns and its absolute value

From the distributed basic statistics of the Shanghai securities composite index, we can know that the skewness is 0.0599893 , which is close to 0. But whether it's zero statistically is still unknown. And the kurtosis is 6.987271. For normal distribution, the skewness is 0 , kurtosis is 3 . On preliminary judgment, it doesn't obey normal distribution, but High Kurtosis and Fat Tail. From the density function diagram of returns (Fig.3), it is more obvious.

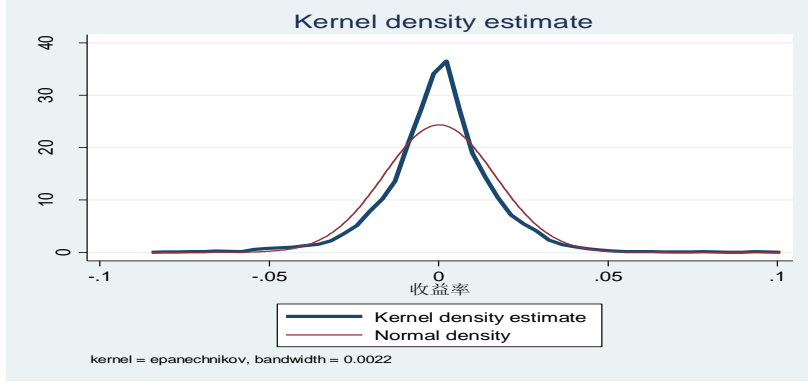

Figure 3. Density function diagram of returns

From the archqq inspection diagram (Fig.4) for sequence diagram of returns, we can find that middle part and tail are obvious deviation. And the test for skewness accepts null hypothesis of normal distribution, while the test for kurtosis refuses null hypothesis and the same with joint test of the two. So from the perspective of statistics, returns time series does not obey normal distribution

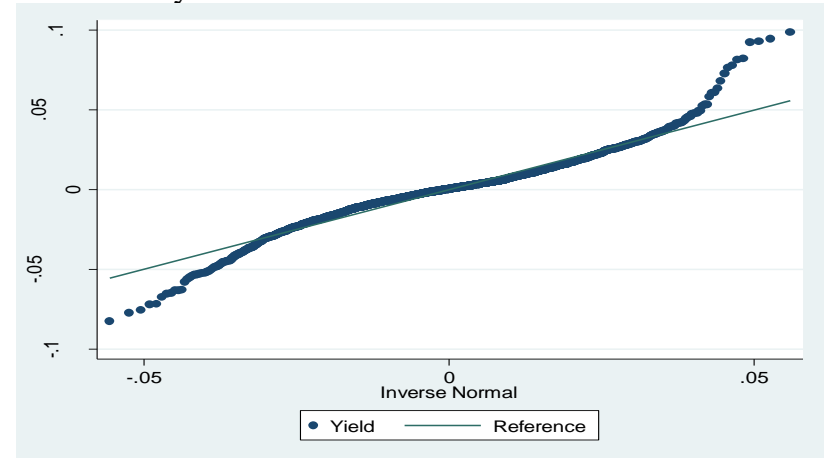

Figure 4. archqq inspection diagram 
From the above, the Shanghai securities composite index returns time-series data really exist the characteristic of High Kurtosis and Fat Tail on statistics, and the diagrams also show volatility clustering. However, whether volatility clustering is statistically significant depends on the existence of ARCH effect.

\section{B. Check Whether There is the ARCH Effect on the Shanghai Securities Composite Index Returns}

Firstly, through the regression of return sequences and constant, do the test of lagging 20 order to check whether there is the ARCH effect. The check results indicate that from lag 1 to 20 order rejected the null hypothesis that there is no ARCH effect. Therefore, it proved that there is $\mathrm{ARCH}$ effect on return sequences.

Even think return lag items affect the current returns, then use returns to do the regression of its lag 3 order to check whether there is ARCH effect. The result is also the same. And there is ARCH effect on return sequences, even adding the lag term in the regression.

\section{Estimation and Testing of the ARCH Model}

Proved there is ARCH effect, then should estimate the ARCH model. Firstly, determine the lag order number. Use graphic method to do the regression of returns and constant and get the square of residuals fitted value e2. Make its autocorrelation figure and partial autocorrelation figure. Despite the graphic result is not ideal, if regard some special high order individual significantly lag items as outlier's effect, the estimation is that the lag order is 8 concisely and the lag order is 10 conservatively. According to the principles of information law, we can make more accurate judgment. Such as the lag order is 20 based on AIC principle and lag order is 12 based on BIC principle. For the simplicity of the model, this paper choose the lag order is 12 . Based on BIC principle, do the ARCH estimation of return sequences and predict conditional variance $g_{-} t$ and residual of mean equation e_t. Make the sequence diagram of predicted conditional variance g_t (Fig.5). We can see the phenomenon of volatility clustering.

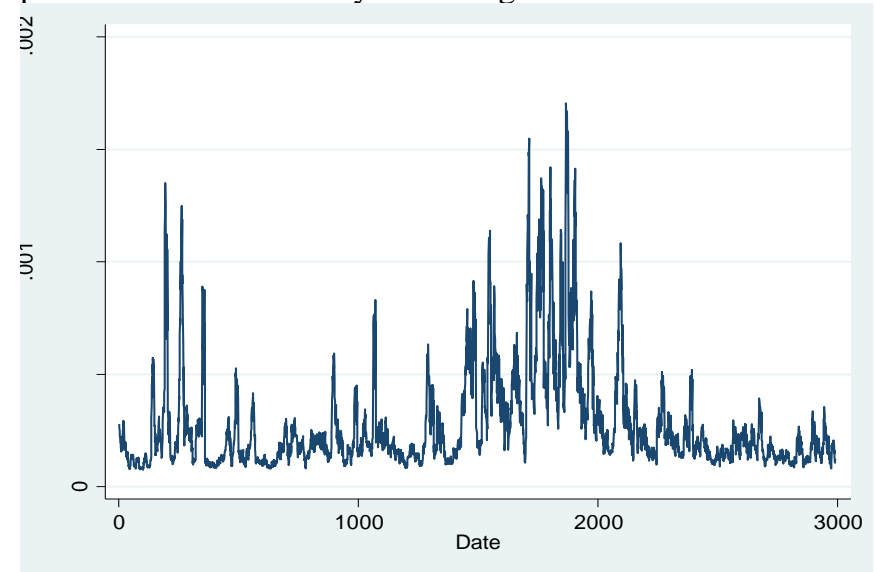

Figure 5. sequence diagram of predicted conditional variance $\mathrm{g} \_\mathrm{t}$

Standardize the residual of the model above to get standardized residual $\mathrm{zt}$ and the square of standardized residual zt2. Check them in order to find out the rationality of ARCH model's mean equation and conditional variance equation. If the mean equation is rational, then standardized residual is a random process of i.i.d, that there is no serial correlation and ARCH effect. If the conditional variance equation is rational, then the square of standardized residual is a random process of i.i.d.

From the partial autocorrelation figure of standardized residual $\mathrm{zt}$, there is obvious 3 order partial autocorrelation, which indicate the existence of serial correlation of $\mathrm{zt}$. And Ljung - Box statistics, at the beginning of lag of 2 order, still rejected the null hypothesis that there is no serial correlation.

From the partial autocorrelation figure of $\mathrm{zt} 2$, we can conclude that there is no obvious serial correlation of $\mathrm{zt} 2$. Except for the 30th order, which can be regarded as the effect of outlier. And the Ljung - Box statistics all accept the null hypothesis that there is no serial correlation from lag 1 order to 40 order. Therefore, there is no obvious serial correlation on $\mathrm{zt} 2$.

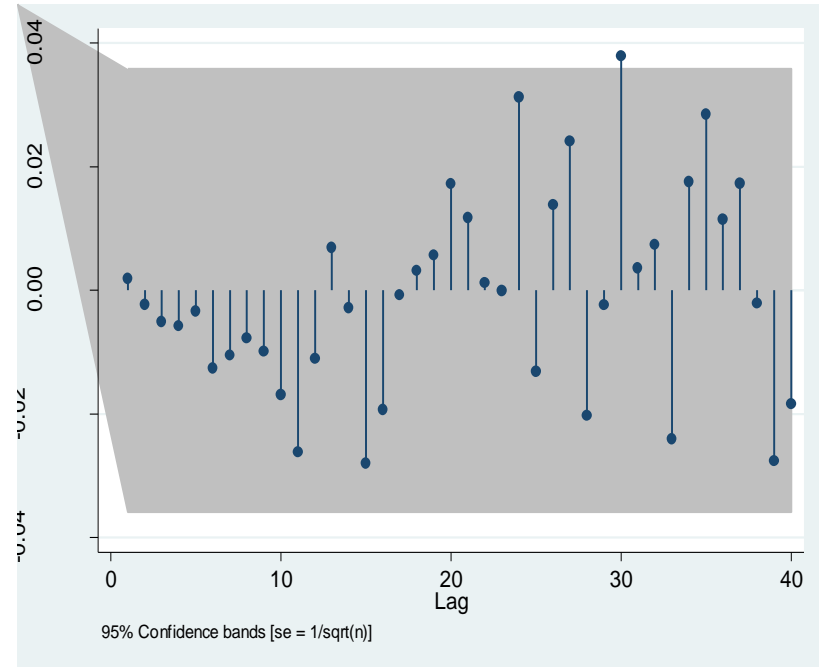

Figure 6. Partial autocorrelation figure of zt2

Do the white noise test on both zt and zt2 (Fig.7 and Fig.8), and we can see they obey the process of i.i.d.

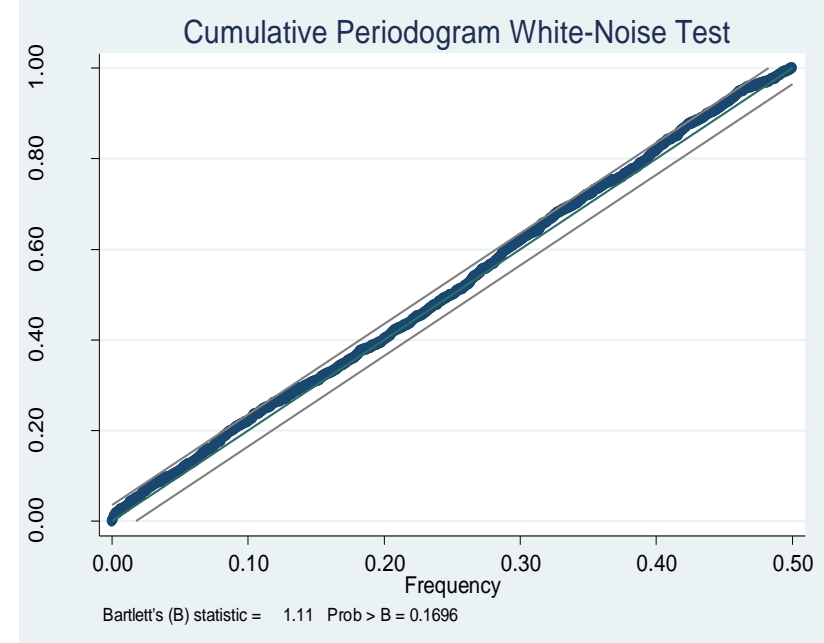

Figure 7. White noise test of zt 


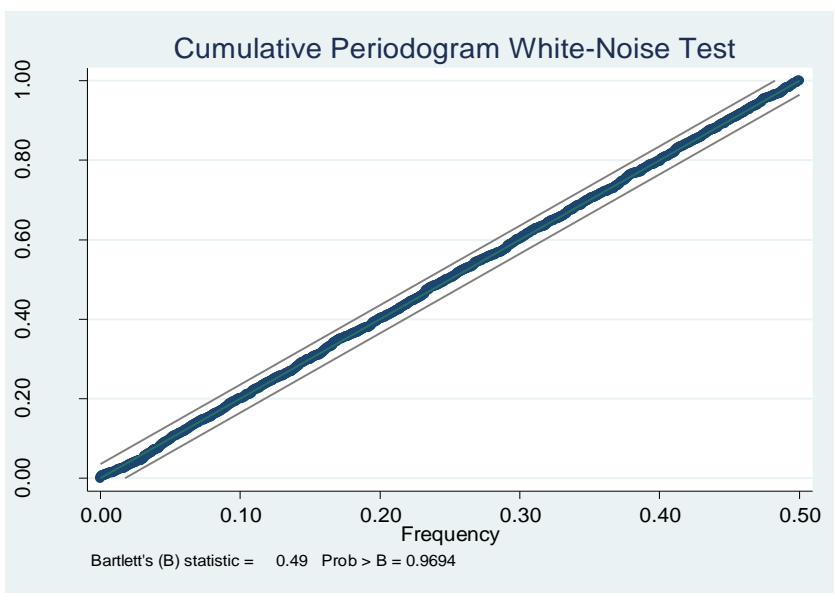

Figure 8. White noise test of $\mathrm{zt} 2$

By the above analysis, for the mean equation, standardized residual zt exists serial correlation, but it also obeys the process of i.i.d based on the white noise test. The mean equation needs to be improved. For the conditional variance equation, there is no serial correlation on the square of standardized residual $\mathrm{zt} 2$, and it obeys the process of i.i.d based on the white noise test. So the conditional variance equation is more reasonable.

\section{GARCH Analysis of the Shanghai Securities Composite Index}

Generally, low order GARCH model is superior to high order GARCH model. In order to verify the case, do the $\mathrm{GARCH}(1,1), \mathrm{ARCH}$ ( 12 ) and ARCH ( 20 ) estimation of the returns of Shanghai securities composite index. The conclusion is that $\operatorname{GARCH}(1,1)$ is superior to ARCH (12) and ARCH (20) based on principles of AIC and BIC.

GARCH $(1,1)$--t-distribution

Both estimates of the ARCH model and GRACH (1.1) model did before suppose that the residuals of mean equation obey normal distribution. Due to the characteristic of High Kurtosis and Fat Tail, it seems that it obeys t-distribution, which is more reasonable. To verify the case, use GARCH $(1,1)$ model to estimate and use likelihood ratio test. The result rejected the null hypothesis that two results does not exist significant differences. So the GARCH $(1,1)$ which obeys the t-distribution is more reasonable. In addition, based on either AIC principle or BIC principle, the GARCH $(1,1)$ which obeys the $t$-distribution is more reasonable.

GARCH $(1,1)--$ ged distribution

It means that residuals of mean equation obey generalized exponential distribution. This can describe the characteristic of High Kurtosis and Fat Tail more flexibly. When shape $<2$, the generalized exponential distribution has the fat tail; When shape $>2$, it has thin tail; When shape $=2$, the generalized exponential distribution translates into normal distribution. From the regression of GARCH $(1,1)--$ ged distribution, we know the shape $=1.205785<2$, that is to say the residuals indeed exist the characteristic of High
Kurtosis and Fat Tail. In addition, GARCH $(1,1)$ - ged distribution is superior to GARCH $(1,1)-\mathrm{t}$ distribution based on either AIC principle or BIC principle.

\section{E. Garch (1,1)-M Model}

Generally, most financial assets has characteristics of high risk and high reward. To verify whether the returns of Shanghai securities composite index exist this characteristic, GARCH $(1,1)-M$ model's estimation is made for return time series. Result shows that the coefficient of sigma 2 is significantly positive at $5 \%$ level. That is return time series exists the characteristic of high risk and high reward. Besides, the residual square $g_{-} t$ has another two forms $\operatorname{sqrt}\left(g_{-} t\right)$ and $\log \left(g_{-} t\right)$, and the result is the same in any form of three. In addition, g_t in the form of $\log \left(g \_t\right)$ is better based on either AIC principle or BIC principle.

\section{F. Asymmetric GARCH}

To verify whether the variation of returns of Shanghai securities composite index exists asymmetric effect, make an estimation of E-GARCH $(1,1)$ model for it. And the delta= 0.0373076 , which proved that it exists significantly asymmetric effect. The variance equation is: $\log \left(g_{-} t\right)=-$ $0.136+0.983 * \log \left(\mathrm{g} \_\mathrm{t}-1\right)+0.171\left|\mathrm{e} \_\mathrm{t}-1\right|-0.037 * \mathrm{e}_{-} \mathrm{t}-1$

In order to reflect the asymmetric effect more visually, make the news impact curve of E-GARCH $(1,1)$ and residuals of mean equation that obey t-distribution and geddistribution (Fig9 to Fig11). we can see that fluctuations caused by negative interference is significantly higher than that caused by the positive interference, which means obvious information asymmetric effect.

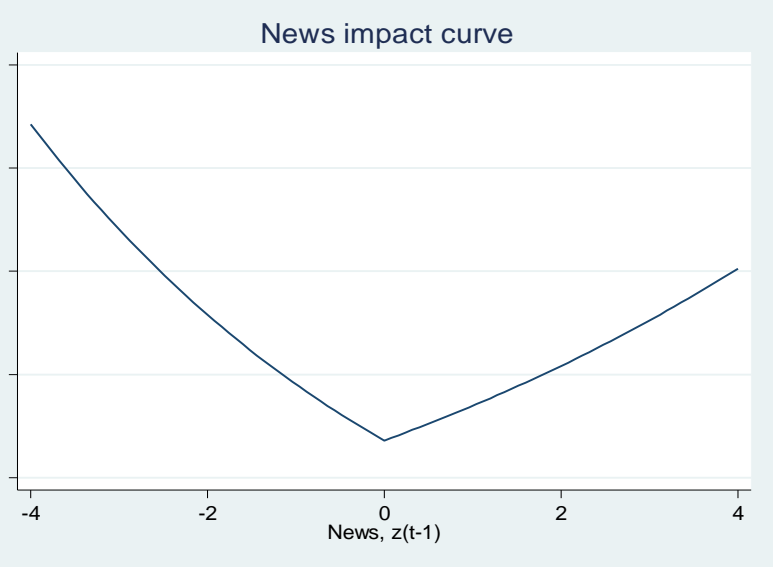

Figure 9. News impact curve of E-GARCH $(1,1)$ model 


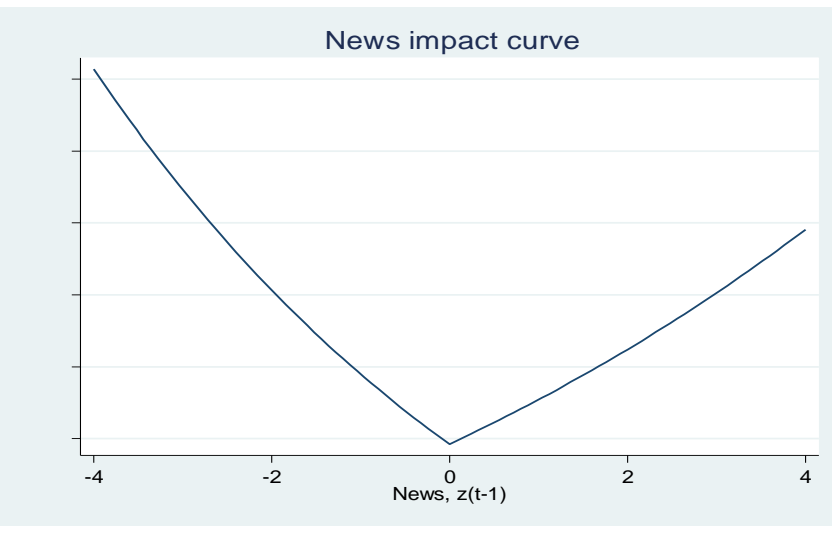

Figure 10. News impact curve of E-GARCH(1,1) - $\mathrm{t}$-distribution model

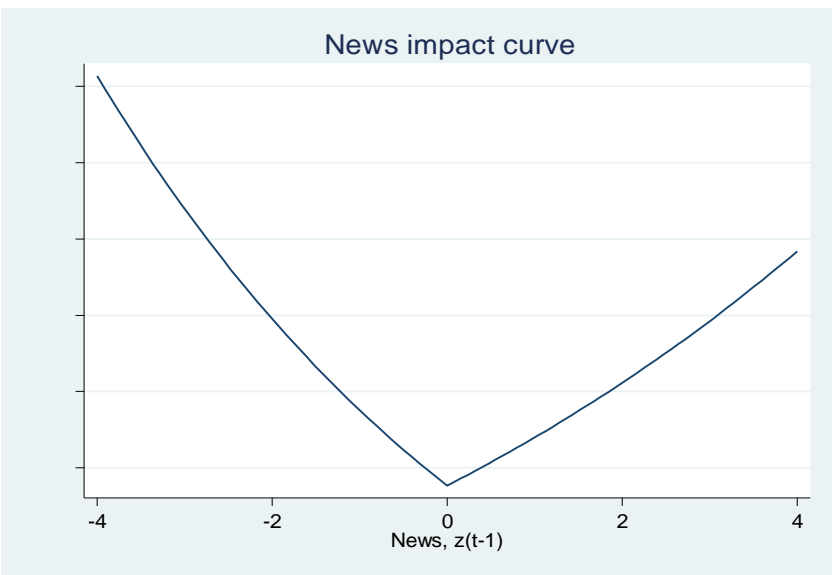

Figure 11. News impact curve of E-GARCH(1,1) — ged-distribution model

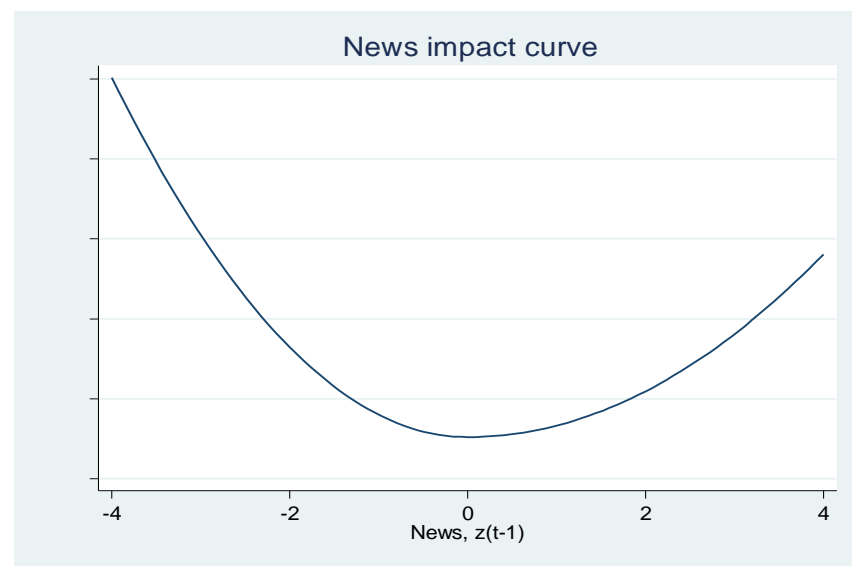

Figure 12. News impact curve of T-GARCH (1,1) model

If make the T-GARCH( 1,1$)$ model estimation for return sequences, result is $\mathrm{g} 1=-0.051$, which means negative significantly. That illustrates fluctuations caused by "bad news" is significantly larger than that caused by "good news". As the news impact curve shows below (Fig12).

\section{G. I-GARCH}

In most cases, in the model of GARCH, the summation of the coefficient of ARCH and the coefficient of GARCH is very closed to 1 . So generate I-GARCH model, which has the characteristic of long-term memory, and estimate it with addition constraint condition: the coefficient of $\mathrm{ARCH}+$ the coefficient of GARCH = 1.Comparing the result with GARCH $(1,1)$ model's, the I-GARCH model is not as good as $\operatorname{GARCH}(1,1)$ model based on either AIC principle or BIC principle.

\section{CONCLUSION}

This paper's study object is the returns of Shanghai securities composite index from January 1, 2001 to May 20, 2013, which study on the volatility. Firstly, verify the return sequences of Shanghai securities composite index exist the characteristics of High Kurtosis and Fat Tail and Volatility Clustering. Secondly, verify the return sequences of Shanghai securities composite index exist ARCH effect. Choose the ARCH model of lagging 12 order, estimate it, and then conclude that mean equation needs to be improved while conditional variance equation is reasonable. Thirdly, GARCH estimate for return sequences of Shanghai securities composite index and find that low order GARCH $(1,1)$ is significantly superior to high order ARCH model, GARCH $(1,1)$ model that obeys ged-distribution is significantly superior to $\operatorname{GARCH}(1,1)$ model that obeys t-distribution and normal distribution. Fourthly, GARCH-M model estimate for return sequences to prove there is the phenomenon that high risk and high reward. Fifthly, E-GARCH $(1,1)$ model and T-GARCH $(1,1)$ model estimate for return sequences to find there is significantly asymmetric effect, which means volatility caused by negative interference is larger than that caused by positive interference. Sixthly, in most cases, in the model of GARCH, the summation of the coefficient of $\mathrm{ARCH}$ and the coefficient of GARCH is very closed to 1 . However, the results estimated by I-GARCH $(1,1)$ model with addition constraint condition is not good as the common GARCH $(1,1)$ model.

\section{REFERENCES}

[1] Peng Jing, Liu Jian-feng, Wang Xiao-tian. Empirical analysis of fractal characteristic in stock return. Journal of Math. 2005(05).

[2] Zhang Yu-chun. ARCH model of share yields on China stock market and empirical analysis. Journal of Capital University of Economics and Business. 2006(01).

[3] Tang Qi-ming, Chen Jian. The ARCH effect analysis of China's stock market. Journal of world economy. 2001(03).

[4] Robert F. Engle. An Introduction to the Use of Arch/Garch Models in Applied Econometrics. NYU Working Paper No. FIN-01-030. 2001. 